\title{
Surveillance of acute infectious gastroenteritis (1992- 2009) and food-borne disease outbreaks (1996-2009) in Italy, with a focus on the Piedmont and Lombardy regions
}

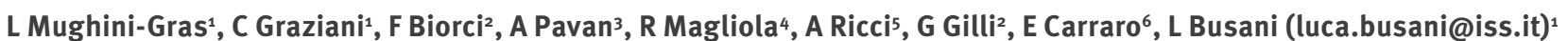

1. Istituto Superiore di Sanità, Department of Veterinary Public Health and Food Safety, Rome, Italy

2. University of Turin, Department of Public Health and Microbiology, Turin, Italy

3. General Public Health Directorate, Unit of Prevention and control of Infectious Diseases, Food Safety and Nutrition, Lombardy region, Milan, Italy

4. Regional reference centre for surveillance, prevention and control of food-borne diseases, Piedmont region, Turin, Italy

5. Istituto Zooprofilattico Sperimentale delle Venezie, Legnaro, Padua, Italy

6. University of Piemonte Orientale 'Amedeo Avogadro', Department of Life Sciences, Alessandria, Italy

Citation style for this article:

Mughini-Gras L, Graziani C, Biorci F, Pavan A, Magliola R, Ricci A, Gilli G, Carraro E, Busani L. Surveillance of acute infectious gastroenteritis (1992-20o9) and foodborne disease outbreaks (1996-2009) in Italy, with a focus on the Piedmont and Lombardy regions.

Euro Surveill. 2012;17(8):pii=20098. Available online: http://www.eurosurveillance.org/ViewArticle.aspx?Articleld=20098

Article published on 23 February 2012

We describe trends in the occurrence of acute infectious gastroenteritis (1992 to 2009) and food-borne disease outbreaks (1996 to 2009) in Italy. In 2002, the Piedmont region implemented a surveillance system for early detection and control of food-borne disease outbreaks; in 2004, the Lombardy region implemented a system for surveillance of all notifiable human infectious diseases. Both systems are internet based. We compared the regional figures with the national mean using official notification data provided by the National Infectious Diseases Notification System (SIMI) and the National Institute of Statistics (ISTAT), in order to provide additional information about the epidemiology of these diseases in Italy. When compared with the national mean, data from the two regional systems showed a significant increase in notification rates of non-typhoid salmonellosis and infectious diarrhoea other than non-typhoid salmonellosis, but for foodborne disease outbreaks, the increase was not statistically significant. Although the two regional systems have different objectives and structures, they showed improved sensitivity regarding notification of cases of acute infectious gastroenteritis and, to a lesser extent, food-borne disease outbreaks, and thus provide a more complete picture of the epidemiology of these diseases in Italy.

\section{Introduction}

Acute gastroenteritis of infectious aetiology is a public health problem worldwide [1]. Although cases in industrialised countries are usually characterised by low mortality, the economic impact on health services (direct costs) and on the general public (indirect costs) can be considerable [2]. Any initiative aimed at controlling acute infectious gastroenteritis in a population should be based on the extent of the problem. However, the true incidence of the disease in the population, based on data from national surveillance systems, is usually underestimated [e.g. 3]. In Italy and other countries, this problem can be attributed to several factors: (i) most cases have mild, self-limiting symptoms, which do not motivate patients to seek medical attention; (ii) stool examination is not always recommended by the attending physician and an aetiological diagnosis is rarely made; (iii) diagnostic capabilities and protocols differ greatly among laboratories; and (iv) underreporting, as it is known that physicians rarely report cases.

In Italy, surveillance of acute infectious gastroenteritis and food-borne disease outbreaks is part of the activities of the Italian National Surveillance System of Infectious Diseases (SIMI), which has been in place since 1990 [4]. Notification data of cases of acute infectious gastroenteritis and food-borne disease outbreaks are also shared with the National Institute of Statistics (ISTAT), which produces official statistics on economic, social and health matters in Italy. The Piedmont and Lombardy regions, in the north of the country, have implemented two different Internetbased surveillance systems since 2002 and 2004, respectively. The Piedmont system is dedicated to surveillance of food-borne diseases, with an emphasis on outbreaks (including but not limited to acute infectious gastroenteritis, as this can frequently be caused by food-borne pathogens), whereas the Lombardy system is aimed at improving the surveillance and reporting of all notifiable human infectious diseases, including 
acute infectious gastroenteritis and food-borne diseases. Both systems notify to the national surveillance system. As the two regions together account for about a quarter of the Italian population (in 2009: Piedmont: 4,432,571 inhabitants; Lombardy: 9,742,676; national: $60,045,068$ [5]) estimates of disease incidence from these regional surveillance systems can be considered relevant for comparisons at the national level.

At present, the national surveillance system does not collect notifications of acute infectious gastroenteritis as one syndrome; instead, laboratory-confirmed cases of diarrhoeal disease are generally notified in two categories: non-typhoid salmonellosis (hereafter referred to as salmonellosis) and infectious diarrhoea other than salmonellosis (hereafter referred to as infectious diarrhoea). These two categories therefore include diarrhoeal diseases caused by all identified enteric pathogens. For the purposes of this article, the official notifications of salmonellosis and infectious diarrhoea were used as proxies for acute infectious gastroenteritis, but we analysed the data separately due to the large difference in the number of cases in the two categories.

Cases of salmonellosis and infectious diarrhoea are notified to the national surveillance system according to its criteria, which, for these diseases, are based on laboratory results [4]. Food-borne disease outbreaks are generally notified to the system as the occurrence of the same disease in two or more people belonging to the same community (family, school, etc.) or exposed to a common source of infection.

The aim of our analysis was to describe the epidemiology of acute infectious gastroenteritis and food-borne disease outbreaks in Italy using official notification data collected in 1992-2009 and 1996-2009, respectively. We have also taken into account the contribution of the notification data from Piedmont and Lombardy and speculated on the impact that the notifications from the two regions could have at the national level. Our findings may help decision-makers in developing novel approaches aimed at improving the surveillance of acute infectious gastroenteritis and food-borne disease outbreaks in the general population.

\section{Methods}

Data collection

Notification data were obtained from the SIMI online databases from 1996 to 2009 (for salmonellosis, infectious diarrhoea and food-borne disease outbreaks) [6] and the ISTAT from 1992 to 1995 (for salmonellosis and infectious diarrhoea) [7]. Data are available on request.

The SIMI started publishing data in 1996, while data of the previous four years were made available by the ISTAT only. There were no available data on food-borne disease outbreaks before 1996. Data on salmonellosis and infectious diarrhoea were collected per year, region, age group (0-14 years, 15-24 years, 25-64 years, 65 years and older) and sex, while those on food-borne disease outbreaks were only available per year and region. Population data per year, region, age group and sex were also collected from the ISTAT.

In order to obtain information on the two regional surveillance systems, we developed a questionnaire according to guidelines provided by the United States Centers for Disease Control and Prevention [8]. The questionnaire is available on request. It was completed by the heads of the two systems.

\section{Data analysis}

Annual notification rates (annual number of notified episodes per 100,000 inhabitants) of salmonellosis and infectious diarrhoea (from 1992 to 2009) were calculated per region, age group and sex, while those of food-borne disease outbreaks (from 1996 to 2009) were calculated per region only. Age- and sex-standardised annual notification rates of salmonellosis and infectious diarrhoea were then calculated per region using 2001 population data. Rates were calculated for the Piedmont and Lombardy regions and for the country as a whole (calculated as the mean of the 20 Italian regions).

Temporal trends in annual notification rates of salmonellosis, infectious diarrhoea and outbreaks of foodborne diseases were assessed using the Cuzick test [9]. Annual rates of salmonellosis and infectious diarrhoea were compared between the sexes using the Mann-Whitney test and among age groups using the Kruskal-Wallis test. Post hoc paired comparisons after the Kruskal-Wallis test were tested using the MannWhitney test on each pair of age group and $p$-value adjustment according to Bonferroni's method [10].

To evaluate any difference in notification rates in Piedmont and Lombardy, compared with the national mean, the standardised annual notification rates of salmonellosis, infectious diarrhoea and food-borne disease outbreaks in both regions were centred on (i.e. subtracted from) the corresponding national mean and then intra-regionally compared between the periods before (Piedmont: 1992 or 1996 to 2001; Lombardy: 1992 or 1996 to 2003) and after the implementation of their respective systems (Piedmont: 2002-2009; Lombardy: 2004-2009), using the Mann-Whitney test.

Statistical analysis was performed with STATA 10.1 and Excel. Statistical significance was set at a $p$ value of 0.05 .

\section{Regional surveillance systems}

All regions other than Lombardy notify cases according to the SIMI criteria [4]. Cases notified to SIMI are not divided into possible, probable or confirmed cases, as in the European Union (EU) case definition [11]. The cases notified to the SIMI are later reported to the EU by the Ministry of Health through the European Surveillance System (TESSy). In contrast, Lombardy, 
uses the EU case definition, but the cases are then reported to the national surveillance system according to SIMI criteria.

\section{Piedmont}

The surveillance system of Piedmont is structurally independent of the SIMI. It collects data on all foodborne diseases - including episodes due to foodpoisoning (e.g. those involving mushrooms, marine biotoxins and histamine) that are not notified to the SIMI. Basically, it is a passive system focused on the early detection of food-borne disease outbreaks, with the aim of improving the rapid alert and investigation of the outbreaks to prevent further cases.

Data generated from the system are also used for: (i) monitoring of spatio-temporal trends in food-borne diseases, including identification of pathogens, food items involved, related risk factors and the at-risk population; (ii) driving the development and evaluation of control programmes (for prioritising resource allocation); (iii) detecting changes in the impact of acute gastroenteritis in response to public health actions; and (iv) providing a basis for epidemiological research.

The system collects information on food-borne disease outbreaks and laboratory-confirmed individual cases of food-borne diseases, thus including salmonellosis and other diarrhoeal pathogens, which are frequently transmitted by contaminated food (Figure 1). Reporting of food-borne diseases is managed separately from other diseases. Each local health unit in the region has dedicated staff who manually enter the received data (usually by fax, email or telephone) into an Internetbased database shared by local health units and the regional health authority. Entry of all validated data is performed on a weekly basis. One person in each local health unit is in charge of validating the data, ensuring that the data are entered and coordinating a multidisciplinary panel of experts to investigate every outbreak of food-borne diseases detected by the system. In the local health unit in the city of Turin, there is a regional coordinator who is in charge of coordinating all other local health units and report to the regional health authority.

\section{Lombardy}

The surveillance system of Lombardy represents an Internet-based improvement of the SIMI and it is fully integrated with it. The system has primarily been implemented to improve aetiological diagnosis and data quality for individual cases. Its main objective is to provide data for real-time analyses on spatio-temporal trends aimed at preventing secondary cases by means of prompt public health actions.

\section{FIGURE 1}

Surveillance system of Piedmont region, Italy

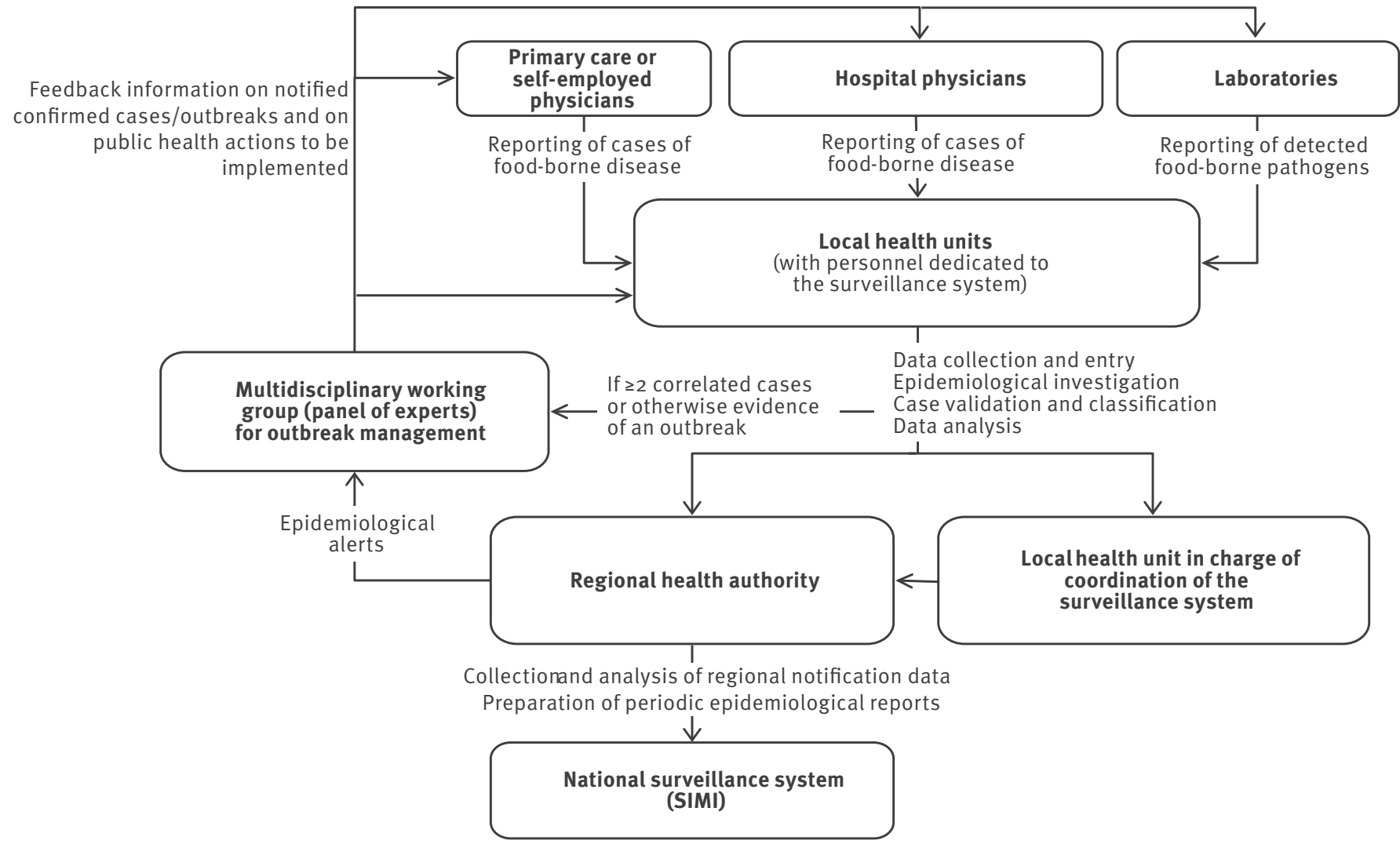


The structure of the Lombardy system (Figure 2) is basically the same as that of the SIMI, which has a pyramidal structure from the bottom (physicians) to the top (regional health authorities) and finally to the Ministry of Health, which hosts the SIMI, but compared with the SIMI, the procedure for physicians reporting to local health units was modified by: (i) reducing the information requested to a minimum (additional information requested by the SIMI for completing the notification is provided by the local health units later on); (ii) shortening the deadline for reporting (e.g. for acute infectious gastroenteritis, notification of cases should be immediate instead of within 48 hours, as required by Italian law) [4]; and (iii) defining different levels of detail required for cases detected at hospitals and for those detected by primary care or self-employed physicians. Data of the notified cases received by each local health unit are manually entered into an Internet-based database and automatically matched with the corresponding patient information stored in the regional health registry. Further epidemiological investigations are carried out when necessary. Cases are automatically validated and classified as notifiable to the SIMI or not notifiable. The database is shared among all local health units and the Lombardy regional health authority, which is in charge of the final data cleaning and analysis.

In both systems, access to the database is restricted to authorised staff of the local health units and regional health authority. All data are managed according to Italian legislation on privacy.

Both systems regularly notify to the SIMI only those cases (divided into salmonellosis and infectious diarrhoea) and food-borne outbreaks that meet the SIMI notification criteria (the set of information that must be collected in order to notify the case to the system is described in the legislation [4]).

\section{Epidemiology of acute infectious gastroenteritis}

and food-borne disease outbreaks in Italy

During the period analysed (1992-2009 for salmonellosis and infectious diarrhoea and 1996-2009 for foodborne disease outbreaks), a total of 222,277 cases of salmonellosis, 46,903 cases of infectious diarrhoea and 7,937 food-borne disease outbreaks were notified in Italy. Piedmont notified 16,431 cases of salmonellosis $(7.4 \%$ of the total), 4,012 cases of infectious diarrhoea (8.6\%), and 570 food-borne disease outbreaks (7.2\%), while Lombardy notified 43,040 cases of salmonellosis (19.4\%), 14,797 cases of infectious diarrhoea (31.5\%), and 1,663 food-borne disease outbreaks $(21.0 \%)$. Annual notification rates of salmonellosis, infectious diarrhoea and food-borne disease outbreaks in Piedmont and Lombardy, together with the national mean, are shown in Figure 3.

\section{FIGURE 2}

Surveillance system of Lombardy region, Italy

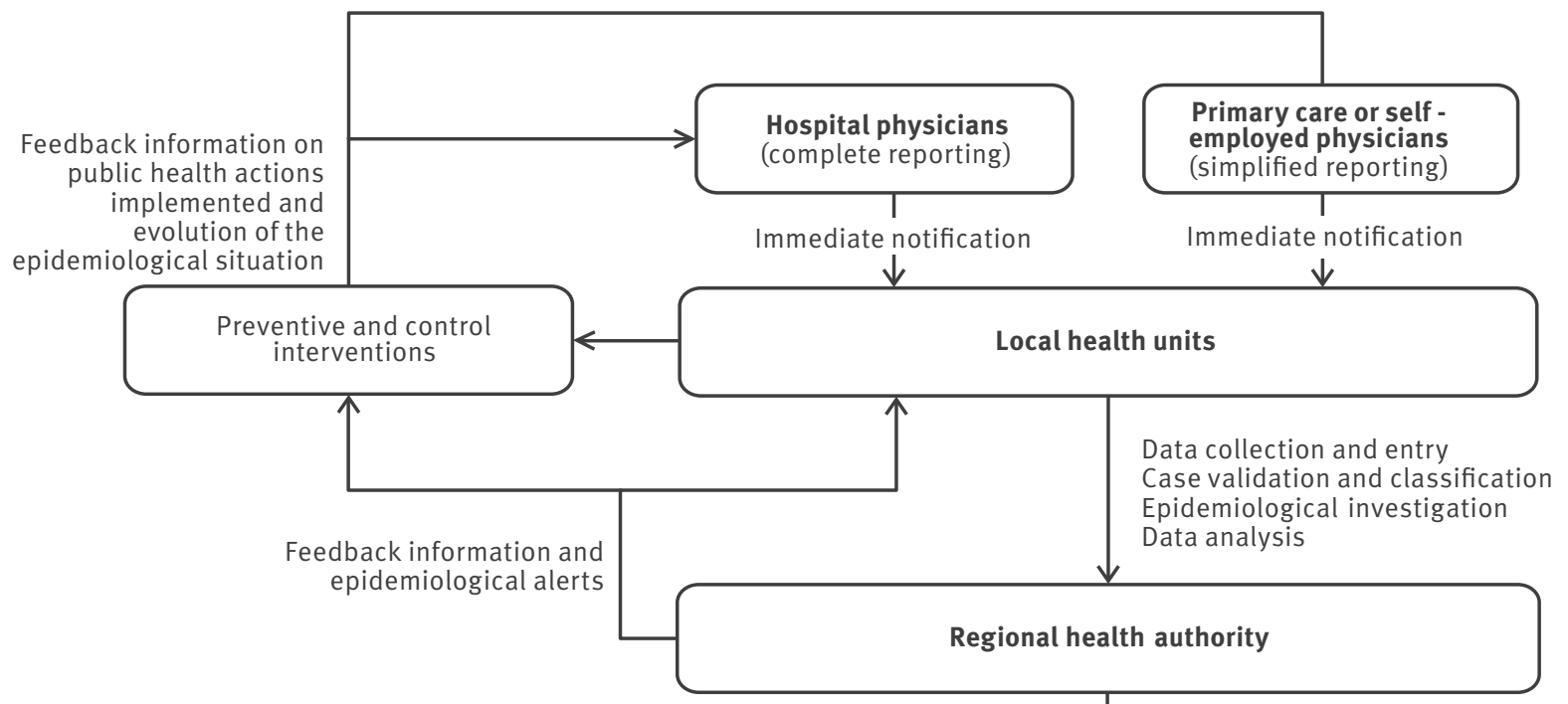

Collection and analysis of regional notification data Preparation of periodic epidemiological reports

National surveillance system (SIMI) 
Salmonellosis notifications

At the national level, salmonellosis notification rates significantly decreased from 47.3 per 100,000 population in 1992 to 6.7 per 100,000 population in 2009 (a decrease of $86 \%$ ). Statistically significant decreasing trends were also observed in Lombardy $(-58 \%$, from 46.2 per 100,000 population in 1992 to 19.5 per $100,000$ population in 2009$)$ and Piedmont $(-82 \%$, from 47.4 per 100,000 population in 1992 to 8.6 per 100,000 population in 2009).

\section{Infectious diarrhoea notifications}

National notification rates of infectious diarrhoea increased significantly from 2.7 per 100,000 population in 1992 to 5.8 in 2009 (an increase of 53\%). From 1992 to 2009, the annual notification rates in Piedmont

\section{FIGURE 3}

Trends of annual notification rates of (A) non-typhoid salmonellosis (1992-2009), (B) infectious diarrhoea other than nontyphoid salmonellosis (1992-2009) and (C) food-borne disease outbreaks (1996-2009) in Piedmont and Lombardy regions and the Italian national mean

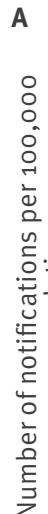

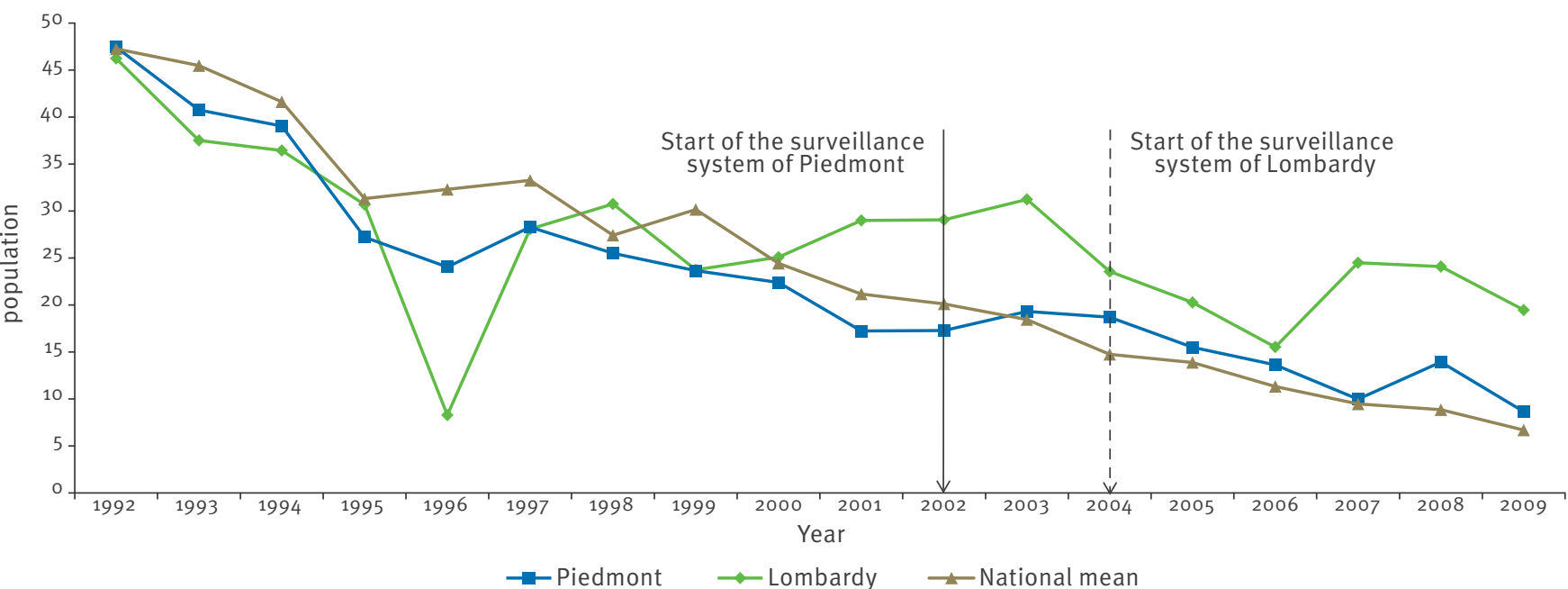

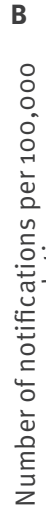

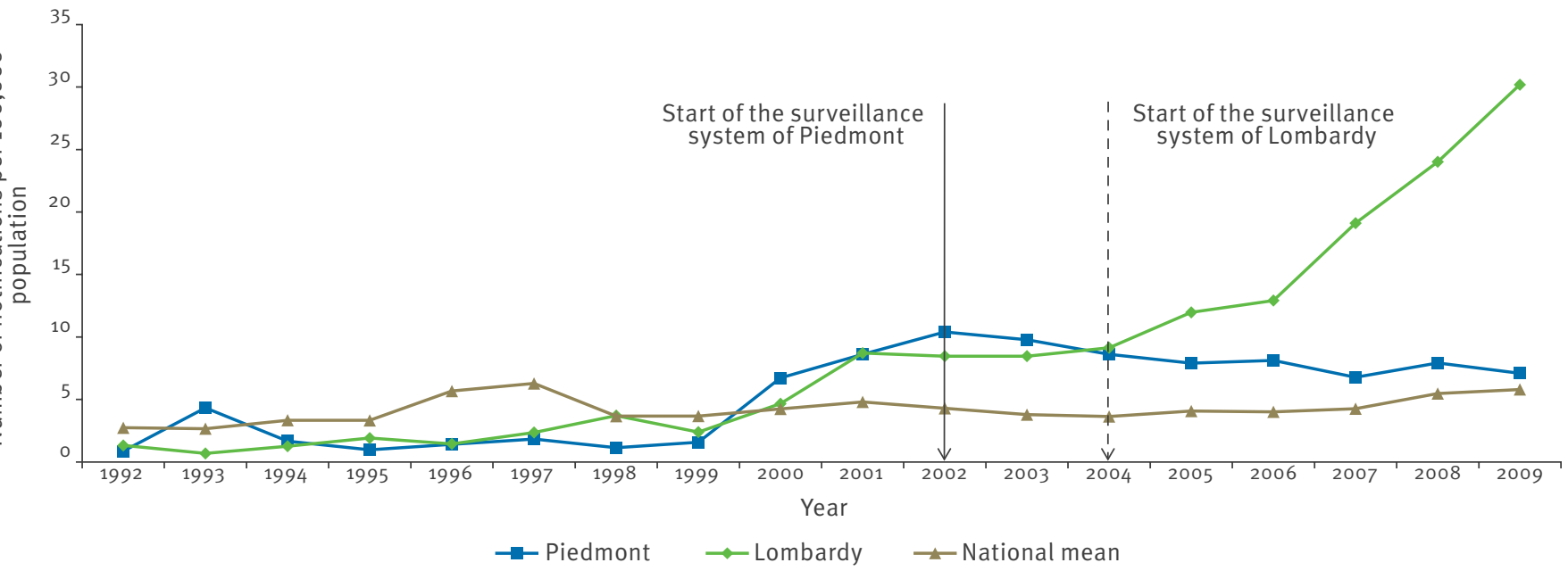

C

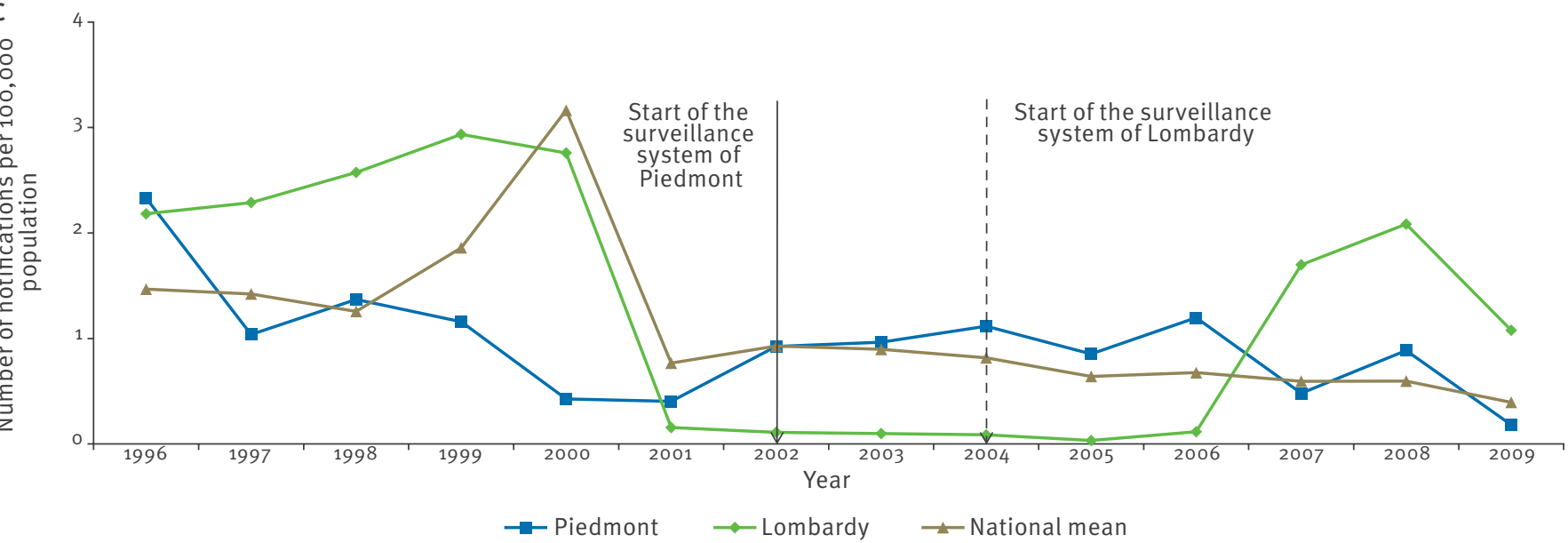


increased significantly from 0.9 per 100,000 population to 7.1 per 100,000 population (+87\%) and from 1.3 per 100,000 population to 30.2 per 100,000 population in Lombardy (+96\%). Figure 3 shows that in both regions, notification rates of infectious diarrhoea were above the national mean from 2000 onwards.

\section{Food-borne disease outbreaks notifications}

The mean national notification rates of food-borne disease outbreaks significantly decreased from 1.5 per 100,000 population in 1996 to 0.4 per 100,000 population in $2009(-73 \%)$. No statistically significant trends were detected in Lombardy $(-50 \%$, from 2.2 per 100,000 population in 1996 to 1.1 per 100,000 population in 2009), where notification rates were below the national mean from 2000 to 2006. From 1996 to 2009 , there was no statistically significant trend in Piedmont, although the notification rate decreased from 2.3 per 100,000 population in 1996 to 0.2 per 100,000 population in 2009 (-91\%). As shown in Figure 3, notification rates were above the national mean from 2003 to 2006 , and then again in 2008, but were below the national mean in 2007 and 2009.

Significant differences in notification rates of salmonellosis and infectious diarrhoea by age group were observed in Piedmont, Lombardy and the country as a whole (Table 1). The highest notification rates were observed in children aged 0-14 years, in both regions and nationally. Apart from the o-14year-olds, the only significant difference was observed in elderly patients ( $\geq 65$ years) in Lombardy for infectious diarrhoea; in this age group the notification rates was 14.10 cases per 100,000 population in Lombardy, while in Italy and in Piedmont the rates were lower (2.84 and 4.36 per 100,000 population, respectively). No statistically significant differences were detected between male and female cases for either salmonellosis or infectious diarrhoea.

Impact of the regional surveillance systems on acute infectious gastroenteritis notification rates

Differences in notification rates from the two regions of salmonellosis, infectious diarrhoea and food-borne disease outbreaks with those of the whole of the country (national mean) before and after the implementation of the regional systems is described in Table 2 . In Piedmont, after implementation of its system, there was a significant increase in notification rates of both salmonellosis (an increase of 1.6 cases per 100,000 population per year) and infectious diarrhoea (an increase of 3.9 per 100,000 population per year) compared with the national mean. In Lombardy, the increase after the implementation of its system was significant for both salmonellosis (an annual increase of 10.3 cases per 100,000 population) and infectious diarrhoea (an annual increase of 13.3 per 100,000 population). The observed increases in the notification rate of foodborne disease outbreaks after the implementation of the two regional systems (annual increases of 0.1 and 0.2 per 100,000 population in Piedmont and Lombardy, respectively) were not statistically significant.

\section{Discussion and conclusions}

Analysis of the notifications of salmonellosis, infectious diarrhoea and food-borne disease outbreaks showed important differences between the figures provided by the regional surveillance systems of Piedmont and Lombardy and those of the national surveillance system. When we compared the regional figures with the national mean, we found significantly higher notification rates of salmonellosis and infectious diarrhoea in the two regions after the implementation of

\section{TABLE 1}

Mean annual notification rates by age group and sex of non-typhoid salmonellosis and infectious diarrhoea other than nontyphoid salmonellosis, Piedmont and Lombardy regions and Italian national mean, 1992-2009

\begin{tabular}{|c|c|c|c|c|c|c|}
\hline \multirow{3}{*}{$\begin{array}{l}\text { Disease, by region } \\
\text { or nationwide }\end{array}$} & \multicolumn{6}{|c|}{ Mean annual notification rate ${ }^{a}$} \\
\hline & \multicolumn{4}{|c|}{ Age group ${ }^{b}$} & \multicolumn{2}{|c|}{ Sex } \\
\hline & $0-14$ years & $15-24$ years & $25-64$ years & $\geq 65$ years & Male & Female \\
\hline \multicolumn{7}{|c|}{ Non-typhoid salmonellosis } \\
\hline Piedmont & $99.73 \pm 6.09 \S$ & $24.06 \pm 8.00 \dagger$ & $21.73 \pm 9.04^{\dagger}$ & $14.92 \pm 2.12 \dagger$ & $41.98 \pm 6.50$ & $38.24 \pm 6.12$ \\
\hline Lombardy & $127.58 \pm 5.9 \S$ & $19.49 \pm 6.13^{\dagger}$ & $19.11 \pm 7.35^{\dagger}$ & $18.11 \pm 1.71 \dagger$ & $48.03 \pm 7.10$ & $44.12 \pm 6.63$ \\
\hline National mean & $98.20 \pm 6.89 \S$ & $32.65 \pm 12.41 \dagger$ & $24.72 \pm 9.93 t$ & $17.33 \pm 2.60 \dagger$ & $44.45 \pm 7.26$ & $42.00 \pm 7.21$ \\
\hline \multicolumn{7}{|c|}{ Infectious diarrhoea other than non-typhoid salmonellosis } \\
\hline Piedmont & $25.80 \pm 3.15 \S$ & $1.36 \pm 0.18 \dagger$ & $1.49 \pm 0.30 t$ & $4.36 \pm 0.67 \dagger$ & $8.83 \pm 1.76$ & $7.68 \pm 1.54$ \\
\hline Lombardy & $32.43 \pm 4.14 \S$ & $2.85 \pm 0.39 \dagger$ & $1.97 \pm 0.30 \dagger$ & $14 \cdot 10 \pm 3 \cdot 77 \ddagger$ & $14.02 \pm 2.62$ & $11.66 \pm 2.26$ \\
\hline National mean & $19.80 \pm 1.04 \S$ & $1.97 \pm 0.06 t$ & $1.22 \pm 0.05^{\dagger}$ & $2.84 \pm 0.51 \dagger$ & $7.04 \pm 1.09$ & $5.88 \pm 0.89$ \\
\hline
\end{tabular}

a Mean number of cases per 100,000 population \pm standard error.

${ }^{b}$ Post hoc paired comparisons of mean annual notification rates between age groups were tested by the Mann-Whitney test. Symbols (§, $\dagger$ and $\ddagger$ ) indicate the results of the pairwise comparisons: in the same row, age groups marked with different symbols are statistically different when compared (Bonferroni-adjusted p<0.05), while the same symbol in the same row indicates no difference between the age groups.

No statistically significant differences between rates in male and female groups were observed (Mann-Whitney test p>o.05). 
their systems. In addition to these increased rates, the absence in these two regions of the significantly decreasing trend in food-borne disease outbreaks observed at the national level can be considered a positive performance of the systems.

The better performance of the two regional systems could be related to increased motivation of those involved (e.g. physicians, epidemiologists, public health professionals and laboratory staff) to report cases of acute infectious gastroenteritis, increased awareness of the disease and better coordination between laboratory and local health unit teams. In both regional systems, the web-based management and sharing of notification data have facilitated the reporting process and improved the completeness of the information collected. Web-based surveillance systems have become increasingly widespread and it is known that they can improve sensitivity [12-14]. Nonetheless, both Italian regional systems have major weaknesses, in particular: (i) limitations in events covered (the Piedmont system is focussed on food-borne diseases only); (ii) limitations in automatic outbreak detection (spatio-temporal clusters); and (iii) data entry is carried out far from the source. Points ii and iii, in particular, are consequences of the lack of realtime data collection and analysis and of the labourintensive activity required by both systems. These two constraints could considerably be balanced out by full electronic reporting and management of notification data.

Concerning the epidemiology of acute infectious gastroenteritis in Italy, we identified a significantly decreasing trend of salmonellosis over the period analysed, which has also been observed in other industrialised countries, possibly resulting from improved Salmonella control measures in the food chain $[15,16]$. Although the national trend is decreasing, salmonellosis rates in Lombardy and Piedmont showed a rise from 2006 and 2007 onwards, respectively. In 2009, data provided to the European Food Safety Authority (EFSA) showed an increase in the number of Salmonella isolates from human cases in Italy of $\mathbf{2 2 . 2 \%}$, compared with those in 2008 (from 3,232 to 4,156 isolates) [16]. This increase was detected one or two years in advance by the surveillance systems of Piedmont and Lombardy (in 2008 and 2007, respectively), but not by the national surveillance system. The difference between our data and those provided to EFSA can be explained by the different sources: our data are the official notification data, while the data provided to EFSA are from Enter-net, a laboratory-based surveillance network for enteric pathogens [17].

In Lombardy, and to a lesser extent in Piedmont, the trend of salmonellosis observed during 2006 to 2009 seems related to the trend seen for food-borne disease outbreaks in the same period. Taking into account that in the EU most of the acute infectious gastroenteritis outbreaks in humans are caused by Salmonella $[15,16]$, we can hypothesise that, at least in Lombardy, improved outbreak detection could have contributed to the increase of salmonellosis cases notified to the system.

The observed trends of infectious diarrhoea notification rates suggest an increasingly prominent role of pathogens other than Salmonella - in particular Campylobacter jejuni - which is the most frequent cause of acute infectious gastroenteritis in the EU $[15,16]$. The increasing trend of infectious diarrhoea was particularly evident in Lombardy, but was also seen in Piedmont, and could be related to the improved routine laboratory capacity for the detection and notification of pathogens other than Salmonella. In both regions, improvement in laboratory capacity (particularly in Lombardy) was implemented at the same time the surveillance systems were introduced. This enabled the regional diagnostic and microbiology laboratories

\section{TABLE 2}

Differences in annual notification rates of non-typhoid salmonellosis, infectious diarrhoea other than non-typhoid salmonellosis, and food-borne disease outbreaks, Piedmont and Lombardy regions with the Italian national mean, before and after implementation of regional surveillance systems

\begin{tabular}{|c|c|c|c|c|c|c|}
\hline \multirow[b]{3}{*}{ Disease } & \multicolumn{6}{|c|}{ Differences in annual notification rate ${ }^{a, b}$} \\
\hline & \multicolumn{3}{|c|}{ Piedmont } & \multicolumn{3}{|c|}{ Lombardy } \\
\hline & $\begin{array}{c}\text { Before } \\
\text { implementation } \\
(1992 / 1996-2001)^{c}\end{array}$ & $\begin{array}{c}\text { After } \\
\text { implementation } \\
(2002-2009)\end{array}$ & $P$ value & $\begin{array}{c}\text { Before } \\
\text { implementation } \\
(1992 / 1996-2003)^{c}\end{array}$ & $\begin{array}{c}\text { After } \\
\text { implementation } \\
(2004-2009)\end{array}$ & $P$ value \\
\hline Non-typhoid salmonellosis & $-4.05 \pm 0.79$ & $+1.58 \pm 0.83$ & $<0.01$ & $-1.54 \pm 2.79$ & $+10.27 \pm 1.87$ & $<0.05$ \\
\hline $\begin{array}{l}\text { Infectious diarrhoea other than } \\
\text { non-typhoid salmonellosis }\end{array}$ & $-1.12 \pm 0.89$ & $+3.90 \pm 0.61$ & $<0.01$ & $-0.25 \pm 0.87$ & $+13.34 \pm 2.95$ & $<0.01$ \\
\hline Food-borne disease outbreaks ${ }^{d}$ & $-0.53 \pm 0.49$ & $+0.13 \pm 0.08$ & $>0.05$ & $+0.16 \pm 0.32$ & $+0.22 \pm 0.40$ & $>0.05$ \\
\hline
\end{tabular}

Mean number of cases per 100,000 population \pm standard error.

Reference value (national mean) $=0$.

From 1992 for salmonellosis and infectious diarrhoea and from 1996 for food-borne disease outbreaks.

d In Piedmont, includes also outbreaks due to food poisoning. 
to extend the range of assays routinely performed and pathogens searched for, and to improve the timeliness of diagnosis and their communication with the staff of the local and regional health authorities involved in the system.

Acute infectious gastroenteritis notification rates by age group confirmed the higher incidence of both salmonellosis and infectious diarrhoea in children (0-14 years), in line with what has been observed in the United States [18] and in other European countries [e.g. 19].

Concerning the trend of food-borne disease outbreaks, Lombardy showed a very low notification rate between 2001 and 2006. This is probably related to the changes in the notification procedure of such outbreaks to the SIMI (but not the notification of single cases) that Lombardy made in 2001, during the period considered for the analyses. After 2006, however, the reporting of these outbreaks was redefined, in agreement with the SIMI definitions.

In Lombardy, we observed that the implementation of the system improved notification rates of acute infectious gastroenteritis and food-borne disease outbreaks, with a reduction of the under-reporting, and consequently gave a better estimate of the impact of acute infectious gastroenteritis on the population. The Piedmont surveillance system, which is dedicated to acute infectious gastroenteritis, allows broader collection of information that is not easy to obtain in other ways, in particular concerning food-poisoning outbreaks.

With regard to the extension of the surveillance systems of Piedmont and/or Lombardy to the other Italian regions, and even to other countries, decisions should be made on the basis of cost-benefit analyses that take into account the expected improvements in terms of efficacy of the surveillance and the resources needed to achieve them, as well as the long-term sustainability of the systems.

In conclusion, improving the surveillance of acute infectious gastroenteritis at the Italian national level requires additional efforts, which can be defined by looking at the experience at the regional level, such as that of Lombardy and Piedmont. Such efforts should be focused on the integration and harmonisation of different surveillance activities and sources of information, as well as evaluation of such activities, to obtain the best achievable impact on the burden of acute infectious gastroenteritis in the population.

\section{Acknowledgments}

This work was supported by the grant from the Italian Ministry of Health - Centro di Controllo delle Malattie 'Stima di base dell'incidenza delle gastroenteriti acute in Italia e integrazione dei dati di notifica, ricovero e laboratorio per la valutazione dell'impatto sulla salute' (2008).
References

1. Kosek M, Bern C, Guerrant RL. The global burden of diarrhoeal disease, as estimated from studies published between 1992 and 2000. Bull World Health Organ. 2003;81(3):197-204.

2. Scallan E, Majowicz SE, Hall G, Banerjee A, Bowman CL, Daly $L$, et al. Prevalence of diarrhoea in the community in Australia, Canada, Ireland, and the United States. Int J Epidemiol. 2005;34(2):454-60.

3. Majowicz SE, Edge VL, Fazil A, McNab WB, Doré KA, Sockett PN, et al. Estimating the under-reporting rate for infectious gastrointestinal illness in Ontario. Can J Public Health. 2005;96(3):178-81

4. Carrieri MP, Salmaso S, Bella A, D’Ancona F, Demicheli $\mathrm{V}$, Marongiu C, et al. Evaluation of the SIMI system, an experimental computerised network for the surveillance of communicable diseases in Italy. Eur J Epidemiol. 2000;16(10):941-7.

5. Italian National Institute of Statistics (ISTAT). Demografia in cifre. Popolazione residente, anno 2009. [Demography in figures. Resident population, year 2009]. ISTAT: Rome. [Accessed 19 Jan 2012]. Italian. Available from: http://demo. istat.it/index_e.html

6. Italian Ministry of Health. Infectious Diseases and Vaccinations. Bollettino epidemiologico. [Epidemiological bulletin]. Italian Ministry of Health: Rome. [Accessed 24 January 2011]. Italian. Available from: http://www.salute.gov. it/malattielnfettive/paginalnternaMenuMalattielnfettive.jsp?id $=812 \&$ menu $=$ strumentieservizi

7. National Institute of Statistics (ISTAT). Health for all - Italia. [Health for all - Italy]. ISTAT: Rome. [Accessed 24 Jan 2011]. Italian. Available from: http://www.istat.it/it/archivio/14562

8. German RR, Lee LM, Horan JM, Milstein RL, Pertowski CA, Waller MN. Updated guidelines for evaluating public health surveillance systems: recommendations from the Guidelines Working Group. MMWR Recomm Rep. 2001;50(RR-13):1-35; quiz CE1-7.

9. Cuzick J. A Wilcoxon-type test for trend. Stat Med. 1985;4(1):87-90.

10. Hollander M, Wolfe DA. Nonparametric statistical methods. 2nd ed. Wiley; 1999.

11. European Commission. Commission Decision of 28 April 2008 amending Decision 2002/253/EC laying down case definitions for reporting communicable diseases to the Community network under Decision No 2119/98/EC of the European Parliament and of the Council. Official Journal of the European Union. Luxembourg: Publications Office of the European Union. 18.6.2008: L 159/46. Available from: http://eur-lex.europa.eu/ LexUriServ/LexUriServ.do?uri=0]:L:2008:159:0046:0090:EN: PDF

12. Ward M, Brandsema P, van Straten E, Bosman A. Electronic reporting improves timeliness and completeness of infectious disease notification, the Netherlands, 2003. Euro Surveill. 2005;10(1):pii=513. Available from: http://www. eurosurveillance.org/ViewArticle.aspx?Articleld $=513$

13. Centers for Disease Control and Prevention (CDC). Progress in improving state and local disease surveillance--United States, 2000-2005. MMWR Morb Mortal Wkly Rep. 2005;54(33):822-5.

14. Rolfhamre P, Jansson A, Arneborn M, Ekdahl K. SmiNet-2: Description of an internet-based surveillance system for communicable diseases in Sweden. Euro Surveill. 2006;11(5): pii=626. Available from: http://www. eurosurveillance.org/ViewArticle.aspx?Articleld $=626$

15. European Centre for Disease Prevention and Control (ECDC). Annual epidemiological report 2011. Reporting on 2009 surveillance data and 2010 epidemic intelligence data. Stockholm: ECDC; 2011.

16. European Food Safety Authority (EFSA), European Centre for Disease Prevention and Control (ECDC). The European Union summary report on trends and sources of zoonoses, zoonotic agents and food-borne outbreaks in 2009. EFSA Journal. 2011;9(3):2090. Available from: http://www.efsa.europa.eu/fr/ efsajournal/pub/2090.htm

17. Busani L, Scavia G, Luzzi I, Caprioli A. Laboratory surveillance for prevention and control of foodborne zoonoses. Ann Ist Super Sanita. 2006;42(4):401-4.

18. Jones TF, McMillian MB, Scallan E, Frenzen PD, Cronquist $A B$, Thomas $S$, et al. A population-based estimate of the substantial burden of diarrhoeal disease in the United States; FoodNet, 1996-2003. Epidemiol Infect. 2007;135(2):293-301.

19. de Wit MA, Koopmans MP, Kortbeek LM, Wannet WJ, Vinjé J, van Leusden F, et al. Sensor, a population-based cohort study on gastroenteritis in the Netherlands: incidence and etiology. Am J Epidemiol. 2001;154(7):666-74. 